\title{
Analysis and Design of Diversity Schemes for Ad Hoc Wireless Networks
}

\author{
Martin Haenggi, Senior Member, IEEE
}

\begin{abstract}
Diversity schemes permit efficient communication over fading channels but are often hard to analyze and design in networks with many nodes. For Rayleigh-fading channels, there exists an interesting relationship between resistive circuits and time and path diversity mechanisms in wireless ad hoc networks. A resistor-like network element, the erristor, representing the normalized noise-to-signal ratio, is introduced. Given an end-to-end packet delivery probability, the logarithmic mapping from link reception probabilities to erristor values greatly simplifies the problems of power allocation and the selection of time and path diversity schemes, which is illustrated in a number of examples. We focus on transmission strategies with selection combining and simple noncoherent "decode-and-forward" strategies, which is motivated by their practicality. Thanks to its conceptual simplicity, the formalism that is developed provides valuable insight into the benefits of diversity mechanisms.
\end{abstract}

Index Terms-Ad hoc networks, diversity methods, Rayleigh channels.

\section{INTRODUCTION}

I $\mathrm{N}$ AD HOC wireless networks, energy and interference considerations often necessitate multihop routing, where nodes also act as routers, forwarding other nodes' packets [1], [2]. Routing schemes that were developed for wired networks will perform suboptimally since they are based on virtually error-free point-to-point links, thereby ignoring two fundamental properties of the wireless link: 1) the fragility of the channel due to fading and interference [2], [3] and 2) the inherent broadcast property of wireless transmissions. ${ }^{1}$ Fading can be an advantage if transmissions can be scheduled opportunistically [4], [5], and the broadcast property can be exploited by transmission schemes that are based on the principle of cooperative diversity [6], where nodes coordinate both direct and relayed transmissions. Cooperative diversity is a form of spatial diversity, which, in the case of static single-antenna nodes, reduces to path diversity. The other promising strategy (in the case of narrowband channels) against fading is time diversity, which, for slow (nonergodic) fading channels and relatively short packets, is mainly exploited in the form of retransmissions.

For the analysis of multihop packets networks, a deterministic "disk model" is often used [7]-[15], where the radius for

Manuscript received October 15, 2003; revised July 16, 2004. This work was supported in part by the Defense Advanced Research Projects Agency (DARPA)/IXO under Grant AF-F30602-01-2-0526 and in part by the National Science Foundation (NSF) under Grant ECS02-25265 and Grant ECS03-29766.

The author is with the Department of Electrical Engineering, University of Notre Dame, Notre Dame, IN 46556 USA (e-mail: mhaenggi@nd.edu).

Digital Object Identifier 10.1109/JSAC.2004.837357

${ }^{1}$ We assume that omnidirectional antennas are employed. a successful transmission has a power-dependent deterministic value, irrespective of the condition of the wireless channel. Interference is commonly taken into account using the same geometric disk abstraction. The stochastic nature of the fading channel and, thus, the fact that the signal-to-noise-and-interference ratio (SINR) is a random variable are neglected. In general, the volatility of the channel cannot be ignored in wireless networks [2], [3], and a rigorous assessment of diversity benefits is not possible with a deterministic model. The inaccuracy of the "prevalent all-or-nothing model," as the disk model is called in [16], has also been pointed out in [17] and is easily demonstrated experimentally [18], [19]. To overcome some of these limitations of the disk model, we employ a Rayleigh-fading link model that relates transmit power, large-scale path loss, and the success of a transmission.

Based on this model, we present a simple yet powerful formalism that allows an efficient analysis and design of time and path diversity strategies with selection combining for Rayleighfading channels. In the analysis problem, the transmit power levels are given and the end-to-end reliability $p_{\mathrm{EE}}$ is to be determined, whereas in the (more interesting) design problem, we assume that the application dictates a certain end-to-end reliability $p_{\mathrm{D}}$, and the question is how to choose the transmit powers, the relays (paths), and the number of transmissions over each link in order to minimize energy consumption and/or maximize network lifetime under the constraint $p_{\mathrm{EE}} \geq P_{\mathrm{D}}$. We focus on noncoherent "decode-and-forward" strategies, which are, in contrast to coherent combining, straightforward to implement. Nonetheless, they exhibit greatly superior performance to schemes without diversity, in particular at high SNR.

The rest of this paper is organized as follows. In Section II, the link model is presented. Section III introduces the "erristor" framework, where transmissions are characterized by a resistorlike circuit element whose value equals the normalized noise-tosignal ratio at the receiver, and discusses its use for multihop routes and time diversity schemes. Section IV focuses on path diversity schemes and presents several examples, and Section V concludes the paper.

\section{LINK MODEL}

We assume a narrowband multipath channel, modeled as a Rayleigh block fading channel [20] with an additive white Gaussian noise (AWGN) process $z$. The received signal at time $k$ is $y_{k}=a_{k} x_{k}+z_{k}$, where $a_{k}$ is the large-scale path loss multiplied by the fading coefficient. The variance of the noise process is denoted by $N_{0}$.

The transmission from node $i$ to node $j$ is successful if the SINR $\gamma$ is above a certain threshold $\Theta$ that is determined by 
the communication hardware and the modulation and coding scheme [2].

With the assumptions above, the received power $Q$ is exponentially distributed with mean $\bar{Q}=P_{0} d^{-\alpha}$, where $P_{0}$ is proportional to the transmit power, ${ }^{2} d=\left\|x_{i}-x_{j}\right\|_{2}$ the distance between transmitter and receiver, and $\alpha$ the large-scale path loss exponent. $I$ is the interference power affecting the transmission, i.e., the sum of the received powers of all the undesired transmitters.

The following theorem shows that for Rayleigh-fading networks, it is possible to analyze noise and interference separately.

Theorem 1: In a Rayleigh-fading network, the reception probability $p_{r}=\mathbb{P}[\gamma \geq \Theta]$ can be factorized into the reception probability of a zero-interference network $p_{r}^{N}$ and the reception probability of a zero-noise network $p_{r}^{I}$ as follows:

$$
p_{r}=\underbrace{\exp \left(-\frac{\Theta N_{0}}{P_{0} d_{0}^{-\alpha}}\right)}_{p_{r}^{N}} \cdot \underbrace{\prod_{i=1}^{k} \frac{1}{1+\Theta \frac{P_{i}}{P_{0}}\left(\frac{d_{0}}{d_{i}}\right)^{\alpha}}}_{p_{r}^{I}}
$$

where $d_{0}$ is the distance of the desired transmitter, $d_{i}, 1 \leq i \leq k$ are the distances of the $k$ interferers, and $P_{i}$ are their transmit power levels.

Proof: Let $Q_{0}$ denote the received power from the desired source and $Q_{i}, i=1, \ldots, k$, the received power from $k$ interferers. All the received powers are exponentially distributed, i.e., $p_{Q_{i}}\left(q_{i}\right)=1 / \bar{Q}_{i} e^{-r_{i} / \bar{Q}_{i}}$, where $\bar{Q}_{i}$ denotes the average received power $\bar{Q}_{i}=P_{i} d_{i}^{-\alpha}$. Conditioned on $I$, the probability of correct reception is simply an exponential, so we have

$$
\begin{aligned}
p_{r}= & \mathbb{E}_{I}\left[\mathbb{P}\left[Q_{0} \geq \Theta\left(I+N_{0}\right) \mid I\right]\right] \\
= & \mathbb{E}_{I}\left[\exp \left(-\frac{\Theta\left(I+N_{0}\right)}{\bar{Q}_{0}}\right)\right] \\
= & \int_{0}^{\infty} \cdots \int_{0}^{\infty} \exp \left(-\frac{\Theta\left(\sum_{i=1}^{k} q_{i}+N_{0}\right)}{\bar{Q}_{0}}\right) \\
& \times \prod_{i=1}^{k} p_{Q_{i}}\left(q_{i}\right) \mathrm{d} q_{1} \cdots \mathrm{d} q_{k}
\end{aligned}
$$

from which (1) follows. ${ }^{3}$

This allows an independent analysis of the effect caused by noise and the effect caused by interference. Since we are concerned with the fundamental energy benefits of diversity, the focus of this paper is the noise, i.e., on the first factor in (1). Clearly, $p_{r}^{I}$ is invariant under (global) power scaling, so it does not give an indication at which power levels to transmit. The results that are derived are directly applicable to the case of light

\footnotetext{
${ }^{2}$ This equation does not hold for very small distances. So, a more accurate model would be $\bar{Q}=P_{0}^{\prime} \cdot\left(d / d_{0}\right)^{-\alpha}$, valid for $d \geq d_{0}$, with $P_{0}^{\prime}$ as the average value at the reference point $d_{0}$, which should be in the far field of the transmit antenna. At $916 \mathrm{MHz}$, for example, the near field may extend up to 3-4 ft (several wavelengths).

${ }^{3} \mathrm{~A}$ similar calculation has been carried out in [21, Appendix] for a network with spreading gain and equal transmit powers for all nodes.
}

traffic $\left(p_{r}^{I} \rightarrow 1\right)$. For the heavy traffic case, a separate interference analysis would have to be carried out, as in [22] and [23], and the resultant $p_{r}^{I}$ would have to be multiplied by the $p_{r}^{N}$ derived here.

In a zero-interference network, the reception probability over a link of distance $d$ at a transmit power $P_{0}$, is given by

$$
p_{r}:=\mathbb{P}\left[\gamma_{N} \geq \Theta\right]=e^{-\frac{\Theta N_{0}}{P_{0} d^{-\alpha}}} .
$$

Solving for $P_{0}$, we get for the necessary transmit power to achieve $p_{r}$

$$
P_{0}=\frac{d^{\alpha} \Theta N_{0}}{-\ln p_{r}}
$$

Note that for $\alpha \rightarrow \infty$, the fading model turns into a disk model. For $d<1$, the reception probability is one, for $d>1$, it is zero. Similarly, for the interference term in (1), if all the interferers are further away than the desired transmitter, i.e., $d_{0}<\min \left\{d_{1}, \ldots, d_{k}\right\}$, reception is guaranteed. Otherwise, reception is impossible.

\section{ERRISTOR REPRESENTATION}

\section{A. Connections Without Retransmission}

Over an $n$-hop connection from node 0 to node $n$ in an ad hoc network, the reception probability (end-to-end reliability) is

$$
p_{\mathrm{EE}}=e^{-\Theta \sum_{i=1}^{n} \frac{1}{\bar{\gamma}_{i}}}
$$

where $\bar{\gamma}_{i}$ denotes the mean SNR at receiver $i$. Let $R$ denote the normalized average noise-to-signal ratio (NSR) at the receiver, i.e., $R:=\Theta / \bar{\gamma}$. We get

$$
-\ln p_{\mathrm{EE}}=\sum_{i=1}^{n} R_{i}=R_{\mathrm{tot}} .
$$

With $p_{\mathrm{D}}$ the desired end-to-end reliability, the condition $p_{\mathrm{EE}} \geq$ $p_{\mathrm{D}}$ translates into the condition that the sum or the series connection ${ }^{4}$ of the NSR values $R_{i}$ is at most $R_{\mathrm{D}}:=-\ln p_{\mathrm{D}}$. So, the individual $R_{i}$ 's can be replaced by an equivalent $R_{\mathrm{tot}}$. For a single link, we have

$$
R=-\ln p_{r} \Longleftrightarrow p_{r}=e^{-R} .
$$

For probabilities close to 1 (or $R \ll 1$ ), the following first-order approximations are accurate

$$
\hat{R}:=1-p_{r} \lesssim R \Longleftrightarrow \hat{p}_{r}:=1-R \lesssim p_{r} .
$$

This approximation shows that for small values, the NSR can be considered equivalent to the packet loss probability. To emphasize this fact and the resistor-like series connection property of the NSR, we denote $R$ as an "erristor" and its value as its "erristance." It follows from (5) that over a multihop connection, the noise accumulates and the packet loss probabilities simply add up.

\footnotetext{
${ }^{4}$ In terms of the SNR values, this corresponds to a parallel connection, which was pointed out in [24].
} 


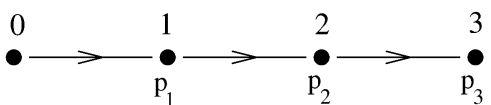

(a)

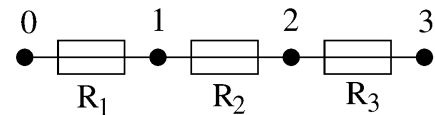

(b)

Fig. 1. (a) Three-hop connection with link reception probabilities $p_{1}, p_{2}$, and $p_{3}$ and the erristor circuit. (b) The erristor values are the normalized noise-to-signal ratios $R_{1}, R_{2}$, and $R_{3} . R_{i}=-\ln p_{i}$.

The relationship between the transmit power and $R$ is

$$
P_{0}=d^{\alpha} \bar{\gamma} N_{0}=\frac{d^{\alpha} \Theta N_{0}}{R}
$$

Henceforth, $P:=d^{\alpha} / R$ denotes the normalized (by $\Theta N_{0}$ ) transmit power.

Example 1: Fig. 1(a) shows an example with three links and their reception probabilities. From (5), we know that a series of hops translates into a series connection of erristors, hence, we find the corresponding erristor network in Fig. 1(b). For $p_{\mathrm{D}}=$ $90 \%$, for example, the value of $R_{1}+R_{2}+R_{3}$ must be at most $R_{D}=-\ln p_{\mathrm{D}} \approx 0.105$. If all the power levels are equal, this can be achieved by setting $R_{1}=R_{2}=R_{3}=0.105 / 3=0.035$. A possible solution with unequal power is $R_{1}=R_{2}=0.05$ and $R_{3}=0.005$. In this case, the probability after two links is $e^{-0.05} e^{-0.05} \approx 90.5 \%$, which is already close to $90 \%$. Consequently, a high amount of energy is consumed at the third link to ensure packet reception with the required probability $e^{-0.005}=99.5 \%$.

If the internode distances $d_{i}$ (between node $i-1$ and node $i$ ) are given, a solution can be determined that ensures that all the transmit power levels have the same value $P$. From $\left(d_{1}^{\alpha}+d_{2}^{\alpha}+\right.$ $\left.d_{3}^{\alpha}\right) / P=-\ln p_{\mathrm{D}}$, we get

$$
P=\frac{d_{1}^{\alpha}+d_{2}^{\alpha}+d_{3}^{\alpha}}{-\ln p_{\mathrm{D}}}
$$

For $d_{i}=i, \alpha=2$, and $p_{\mathrm{D}}=90 \%$, for example, we get $P \approx 14$. $9.5=133$ and $R_{1} \approx 0.0075, R_{2} \approx 0.03$, and $R_{3} \approx 0.0677$.

\section{B. Connections With Time Diversity (Retransmissions)}

In general, with $n$ transmissions over the same link at NSR levels $R_{i}$ and selection combining, the total reception probability is

$$
p_{n}=1-\prod_{i=1}^{n}\left(1-e^{-R_{i}}\right)
$$

To derive a general rule for the simplification of these expressions, we apply the following theorem.

Theorem 2: For $\left(x_{1}, x_{2}, \ldots, x_{n}\right) \in\left(\mathbb{R}_{0}^{+}\right)^{n}$

$$
1-\prod_{i=1}^{n}\left(1-e^{-x_{i}}\right) \geq e^{-\prod_{i=1}^{n} x_{i}}
$$

with equality if and only if $\prod_{i=1}^{n} x_{i}=0$.

The proof is presented in the Appendix.

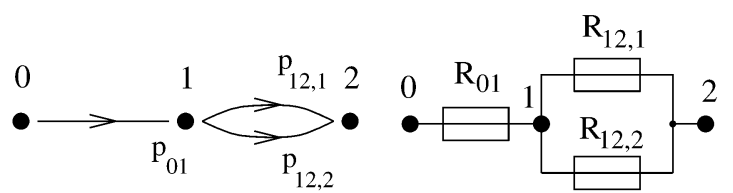

Fig. 2. (Left) A two-hop connection with two transmissions over the second hop. (Right) Corresponding erristor circuit.

So, erristors connected in series behave like regular resistors, whereas the values of erristors connected in parallel (retransmissions) have to be multiplied. Due to the bound (11), the resultant end-to-end reliability will be slightly higher than the one specified.

Example 2: Consider the simple example in Fig. 2. A packet is transmitted with reception probability $p_{01}$ over the first hop and transmitted twice over the second hop, with probabilities $p_{12,1}$ and $p_{12,2}$, respectively. The end-to-end reliability of the connection is $p_{\mathrm{EE}}=p_{01} \cdot\left(1-\left(1-p_{12,1}\right)\left(1-p_{12,2}\right)\right)$. Let $p_{\mathrm{D}}=90 \%$. How to allocate the transmit powers?

For the second link, we have $p_{12}=1-\left(1-p_{12,1}\right)(1-$ $\left.p_{12,2}\right)=1-\left(1-e^{-R_{12,1}}\right)\left(1-e^{-R_{12,2}}\right)$. From Theorem 2 , $e^{-R_{12,1} R_{12,2}}$ is a lower bound for $p_{12}$, and for $R_{1} \ll 1$ and $R_{2} \ll 1$, the bound is tight. Thus, we may replace $R_{12,1}$ and $R_{12,2}$ by $R_{2}=R_{12,1} R_{12,2}$. In the erristor diagram, the two transmissions are illustrated by a parallel connection (see Fig. 2). From $R_{1}+R_{2}<R_{D}=0.1$, a possible choice is $R_{1}=R_{2}=0.05$ and $R_{12,1}=R_{12,2}=\sqrt{5} / 10$. Thanks to the retransmission, node 2 consumes less than half the energy of node 1 , assuming the distances are the same. An allocation where both nodes use the same power is $R_{1}=\sqrt{110} / 5-2 \approx 0.098$, and $R_{12,1}=R_{12,2}=2 R_{1}$. This way, node 1 benefits from node 2 's retransmission and uses almost only half the power.

For $n$ transmissions with the same power level $R$, the difference between the precise probability value $1-\left(1-e^{-R}\right)^{n}$ and the lower bound $e^{-R^{n}}$ is plotted in Fig. 3. The erristance threshold where the bound is within $1 \%$ is $R=0.236$ for $n=2$ and $R=0.375$ for $n=4$. Thus, for $R<1 / 4(p>78 \%)$, the bound is sufficiently tight for all practical purposes. For values $R>1$, the bound is loose, and the multiplication would not make sense, since the overall erristance increases, although, of course, even a retransmission with low power still leads to an improvement in the total link reception probability. However, for $R \gtrsim 1 / 2$, a single transmission outperforms splitting the power into two transmissions. For two transmissions at NSR $2 R$, the reception probability is $p_{r}=1-\left(1-e^{-2 R}\right)^{2}$, whereas for a single transmission at NSR $R$, we get $p_{r}^{\prime}=e^{-R}$. The two probabilities are equal for

$$
R=\ln 2-\ln (\sqrt{5}-1) \approx 0.48
$$

So, for $R \gtrsim 1 / 2$, the reception probability is higher for a single transmission at NSR $R$. From a practical point of view, reception probabilities lower than $37 \%$ may not be worth spending energy at the receiver. By excluding erristances greater than one, this is taken into account, and, at the same time, the validity of the multiplication property is preserved. 


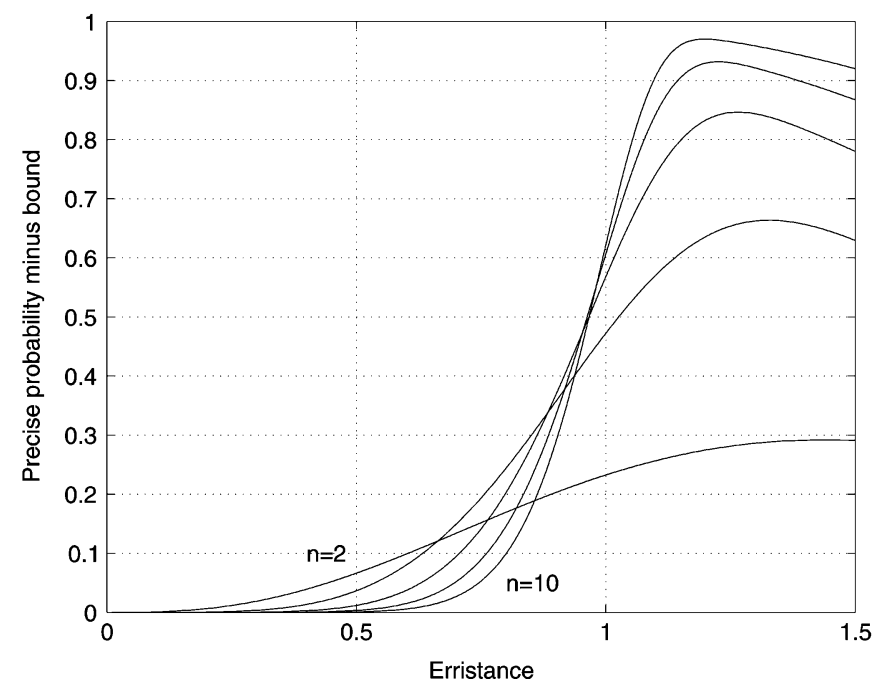

Fig. 3. Difference between the exact probability and the lower bound for $n=$ $2,4,6,8,10$ transmissions with equal erristance $R$.

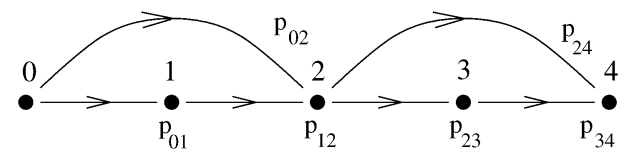

(a)

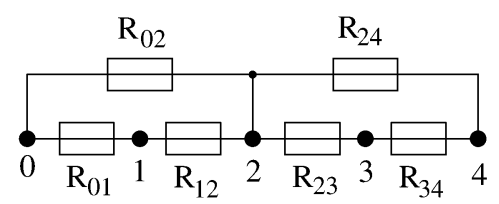

(b)

Fig. 4. (a) Network that exploits path diversity and (b) its erristor circuit.

\section{PATH DiVersity}

\section{A. Transmissions Over Independent Paths}

With the addition and multiplication property of the erristors, we have all the ingredients to analyze and design path diversity schemes.

Example 3: Fig. 4 displays an example of a network, where path independence is guaranteed even when the channels have a long coherence time. By conventional analysis, the end-to-end reception probability is

$$
\begin{aligned}
& p_{\mathrm{EE}}=\left(1-\left(1-e^{-R_{01}} e^{-R_{12}}\right)\left(1-e^{-R_{02}}\right)\right) \\
& \cdot\left(1-\left(1-e^{-R_{23}} e^{-R_{34}}\right)\left(1-e^{-R_{24}}\right)\right) .
\end{aligned}
$$

How to choose the $R_{i j}$ 's to guarantee $p_{\mathrm{EE}} \geq p_{\mathrm{D}}=95 \%$ ? The equivalent erristance [Fig. 4(b)] is $R_{\mathrm{tot}}=\left(R_{01}+R_{12}\right) R_{02}+$ $\left(R_{23}+R_{34}\right) R_{24}$, and $p_{\mathrm{EE}}=e^{-R_{\mathrm{tot}}}$. For $p_{\mathrm{D}}=95 \%$, we have $R_{\text {tot }} \approx 0.05$. Thanks to the symmetry, $\left(R_{01}+R_{12}\right) R_{02}=$ $\left(R_{23}+R_{34}\right) R_{24}=0.025$ is a solution; hence, we may set nearest-neighbor hops to $R_{i-1, i}=0.05$ and $R_{02}=R_{24}=$ 0.25 . Note that the value for the two longer hops is five times bigger, which means that the necessary transmit powers are comparable if the nodes have equal distances and the path loss exponent is between 2 and 3 . So, the diversity scheme and the power allocation guarantee $p_{\mathrm{EE}}=e^{-0.05} \approx 95.1 \%>p_{\mathrm{D}}$. The conventional analysis (13) yields $p_{\mathrm{EE}} \approx 95.8 \%$ which is, as expected, slightly larger. The formalism also permits a rapid

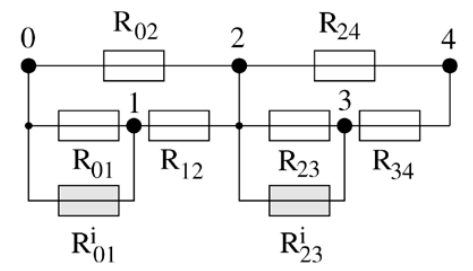

Fig. 5. Erristor circuit for example 3 (see Fig. 4) including implicit transmissions. Implicit erristors are shaded gray.

reallocation of resources, if necessary. Assume node 3 runs out of energy. With $R_{34} \rightarrow \infty$, we see immediately that $R_{23}$ becomes useless, and the only path in the lower half of the diagram will be the one with $R_{24}$. What value does $R_{24}$ need to have to ensure $p_{\mathrm{D}}$ ? Without changing the other erristances, we find $R_{24}=0.025$.

The total normalized energy consumption (per packet) at each node can easily be determined

$$
E_{i}=\sum_{j=1}^{m} \frac{d_{i j}^{\alpha}}{R_{i j}}
$$

where $m$ is the number of outgoing paths from node $i$.

\section{B. Implicit Transmissions}

In example 2 (Fig. 2), if node 2 listens to the transmission from node 0 to node 1 , then this implicit transmission has to be modeled by an additional erristor for an accurate analysis. This implicit erristor is free in terms of transmit power (but still requires power to receive the packet); it represents the benefit from the wireless medium, often denoted as the wireless multicast advantage [2], [25].

Example 2 (Continued): Assume $p_{\mathrm{D}}=99 \%$, so $R_{\mathrm{tot}} \approx$ 0.01 . This is achieved by setting $R_{01}=0.005$ and $R_{12,1}=$ $R_{12,2}=0.07$. However, since there is an implicit transmission from 0 to 2 , there is an erristor in parallel with a value of $R_{02}^{i}=R_{01}\left(d_{02} / d_{01}\right)^{\alpha}$ (the superscript $i$ indicates an implicit transmission). Assuming $d_{02}=2 d_{01}$ and $\alpha=3$, we get $R_{02}^{i}=0.04, R_{\mathrm{tot}}=0.01 \cdot 0.04$, and $p_{\mathrm{EE}} \approx 99.96 \%$, which is much better than the target of $99 \%$. So we can reduce $R_{01}$ to a value that guarantees $\left(R_{01}+0.005\right) R_{01} \cdot 8=0.01$. Solving the resulting quadratic equation yields $R_{01} \approx 1 / 30$, which corresponds to less than $1 / 6$ of the original power.

For large path loss exponents and/or small transmit power levels, the benefit to listeners that are farther away than the intended receiver becomes small, since the implicit erristances will be close to one or even above. However, if the implicit receiver is closer than the intended one or if the transmit power is relatively high, it is worthwhile having the nodes awake and listening.

Example 3 (Continued): In example 3 (Fig. 4), there is an implicit transmission from node 2 to node 3 when node 2 is transmitting to node 4 . If node 3 ignores this transmission, then the previous analysis was correct. If it takes advantage of that information, we have to add another erristor $R_{23}^{i}$, as shown in Fig. 5. Analogously, $R_{01}^{i}$ models the implicit transmission $\overrightarrow{01}$ that occurs due to the explicit transmission $\overrightarrow{02}$.

Assuming equal distances between neighboring nodes, $R_{01}^{i}=2^{-\alpha} R_{02}$ and $R_{23}^{i}=2^{-\alpha} R_{24}$. For $\alpha=2$ and using the 


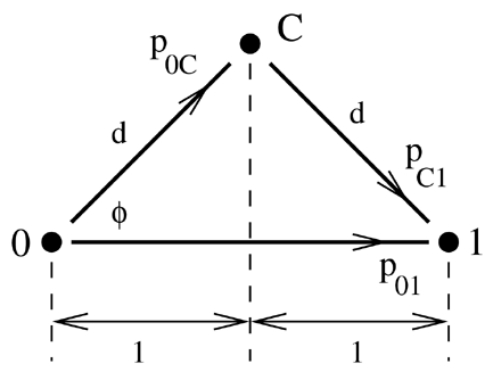

(a)

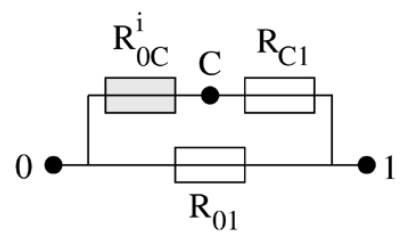

(b)

Fig. 6. (a) Simple cooperative scheme and (b) the corresponding erristor circuit. Node $\mathrm{C}$ is relaying a packet from node 0 to assist node 0 . The distances $\overline{0 C}$ and $\overline{C 1}$ are $d=1 / \cos \phi$.

same values as before, $R_{i, i-1}=0.05$, and $R_{02}=R_{24}=0.25$, we find $R_{01}^{i}=R_{23}^{i}=0.0625$ and

$$
R_{\mathrm{tot}}=2\left(\frac{1}{20} \cdot \frac{1}{16}+\frac{1}{20}\right) \cdot \frac{1}{4} \approx 0.027
$$

resulting in $p_{\mathrm{EE}} \approx 97.3 \%$, which is larger than the target of $95 \%$. Considering that $1 / 16 \ll 1$, we may try to omit the explicit transmission $\overrightarrow{01}$ completely, which results in $R_{\mathrm{tot}}=$ $(1 / 16+1 / 20) / 2 \approx 0.056$ and $p_{\mathrm{EE}}=94.4 \%$. A slight decrease of $R_{02}$ and $R_{24}$ by $10 \%$ each brings $p_{\mathrm{EE}}$ to $95.2 \%$.

The next example demonstrates how the erristor formalism transforms complex logarithmic relationships into simple polynomial ones, which are analytically tractable.

Example 4. A Simple Cooperative Scheme: In Fig. 6, a situation is shown where node 0 wants to transmit to node 1 , and the cooperative node $\mathrm{C}$ may help as a relay. From the erristor circuit, it can be seen that there is no explicit transmission from 0 to $\mathrm{C}$. The goal is to determine which transmission strategy minimizes the total transmit energy $E_{\text {tot }}$ given a certain total erristance $R_{\text {tot }}$.

Let $D:=(d / 2)^{\alpha}$. With $R_{\text {tot }}=R_{01}\left(R_{0 C}^{i}+R_{C 1}\right)$ and $R_{0 C}^{i}=$ $D R_{01}$, we get $R_{\text {tot }}=R_{01}\left(R_{01} D+R_{C 1}\right)$ and $E_{\text {tot }}=2^{\alpha} / R_{01}+$ $d^{\alpha} / R_{C 1}=2^{\alpha}\left(R_{01}^{-1}+D R_{C 1}^{-1}\right)$.

Strategy A-Equal Received Power: A possible strategy is to have $C$ transmit at a power level that makes the received power at node 1 equal to that from the direct transmission $\overrightarrow{01}$, i.e., $R_{C 1}=R_{01}=: R \cdot R_{\mathrm{tot}}$ simplifies to $R_{\mathrm{tot}}=R^{2}(1+D)$ and, thus, $R=\sqrt{\left(R_{\text {tot }}\right) /(1+D)}$. Inserting this expression into the transmit energy $E_{\text {tot }}=\left(2^{\alpha}\right) /(R)(1+D)$ yields

$$
E_{\text {tot }}^{A}=\frac{2^{\alpha}(1+D)^{3 / 2}}{\sqrt{R_{\mathrm{tot}}}} .
$$

Strategy B-Equal Transmit Power: Here, we assume that both node 0 and $\mathrm{C}$ use the same transmit power. With $R:=$ $R_{01}$ and $R_{C 1}=R_{0 C}^{i}=R D$, we have $R_{\mathrm{tot}}=2 D R^{2}$ and

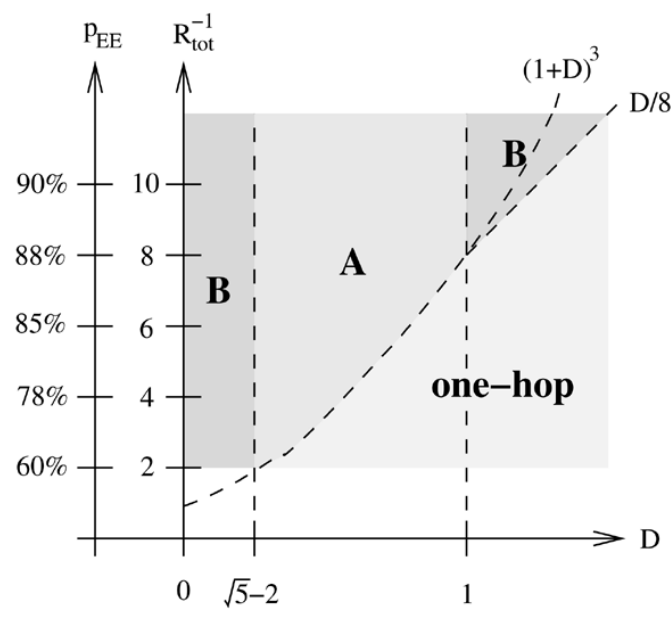

Fig. 7. Visualization of the regions in the $\left(D, R_{\text {tot }}^{-1}\right)$ plane where the different strategies are optimum. Note that the curves $D / 8$ and $(1+D)^{3}$ intersect at the points $\left(\sqrt{5}-2,(\sqrt{5}-1)^{3}\right)$ and $(1,8)$.

$R=\sqrt{\left(R_{\mathrm{tot}}\right) /(2 D)}$. The total energy consumption is simply $E_{\mathrm{tot}}=2 \cdot 2^{\alpha} / R$, or, as a function of $R_{\mathrm{tot}}$

$$
E_{\text {tot }}^{B}=2^{\alpha+1} \sqrt{\frac{2 D}{R_{\text {tot }}}} .
$$

The energy consumption ratio of strategies $\mathrm{A}$ and $\mathrm{B}$ is

$$
\rho:=\frac{E_{\mathrm{tot}}^{B}}{E_{\mathrm{tot}}^{A}}=\frac{2 \sqrt{2 D}}{(1+D)^{3 / 2}} .
$$

$\rho=1$ for $D=1$ and $D=\sqrt{5}-2 \approx 0.236$. For $\sqrt{5}-2<D<$ 1 , strategy A is preferable $(\rho>1)$. The maximum $\rho$, however, is only $(4 / 9) \sqrt{6} \approx 1.089$, occurring at $D=1 / 2$. So, strategy A is at most $8.9 \%$ better.

To get a complete view, we also discuss the case of a direct one-hop transmission and an explicit two-hop scheme without a direct path from 0 to 1 . For the one-hop case, we have $E_{\mathrm{tot}}^{\mathrm{one}}=$ $2^{\alpha} / R_{\text {tot }}$, and for the two-hop case (assuming equal transmit powers), there are two erristors in series with value $R_{\text {tot }} / 2$ and, thus, $E_{\mathrm{tot}}^{\mathrm{two}}=4 d^{\alpha} / R_{\mathrm{tot}}=4 D E_{\mathrm{tot}}^{\mathrm{one}}$.

The one-hop strategy is better if $4 D \geq 1$, or, in terms of the actual distance $d, d>2^{1-2 / \alpha}$. So, for $\alpha=2$ and for $d \geq 2$, one-hop is always better, even for $\alpha \rightarrow \infty$, which is easily explained, since node $C$ is then not closer than node 1 . As a function of the angle $\phi=\arccos (1 / d)$, the condition for one-hop to be better is expressed as $\phi \geq \arccos \left(2^{2 / \alpha-1}\right)$. For $\alpha \rightarrow \infty$, the critical angle is $\phi=\pi / 3$ (corresponding to an equilateral triangle $0 \mathrm{C} 1$ ), as expected.

The last step is the comparison of these simple schemes with the cooperative strategies A and B. First, we note that B always outperforms the two-hop scheme, since it exploits "free" information that is transmitted over the direct path. The tournament between $\mathrm{A}$ and one-hop is won by $\mathrm{A}$ if $R_{\text {tot }}<(1+D)^{-3}$, and B wins against one-hop if $R_{\text {tot }}<(8 / D)$. The resulting division of the $\left(D, R_{\text {tot }}^{-1}\right)$-plane in the different strategies is shown in Fig. 7. Since $R_{\text {tot }}^{-1}$ is related to the SNR, Fig. 7 suggests that cooperation is beneficial at high SNR and relatively small distances $D$, in agreement with intuition.

Example 5. Virtual Antenna Arrays: Several nodes that are close may cooperate and act as a virtual antenna array, exploiting spatial diversity. The performance of such arrays was analyzed in [26] from an information-theoretic perspective. 


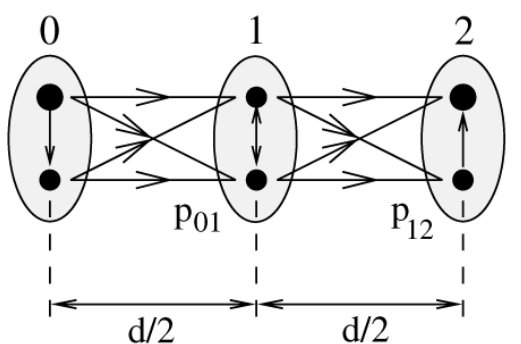

(a)

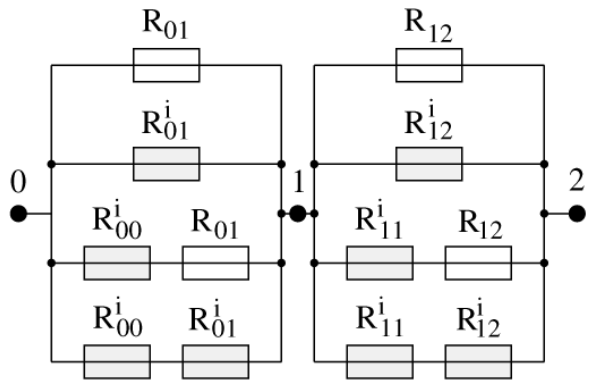

(b)

Fig. 8. (a) Virtual antenna scheme and (b) its erristor circuit.

Here, not assuming any form of lower-layer coordination within the cluster, we apply the erristor formalism to compare these schemes with conventional multihop routing. Fig. 8 shows the erristor diagram of a simple scenario with two nodes assisting each other at the source, in the middle, and at the destination. So, instead of individual nodes, we have clusters of two nodes at positions 0,1 , and 2 . It is assumed that the intracluster distances are much smaller than the intercluster distances $d / 2$. When the source node in cluster 0 is transmitting to cluster 1 , his peer will receive that packet with probability (almost) one since $R_{00}^{i} \ll R_{01}$. In the next time slot, this peer node will transmit the same packet to cluster 1 . Hence, the same packet is delivered over four different paths. Similarly, cluster 1 relays the packet to cluster 2 over four paths. In the case that the actual destination node itself in cluster 2 does not correctly receive the packet, an additional short intracluster transmission is required, whose energy is neglected in the following analysis.

We assume $R_{01}=R_{12}:=R$. For the diversity scheme, with $R_{00}^{i} \ll R$, we get $R_{\text {tot }}=2 R^{4}$ and

$$
E_{\text {tot }}=4 \frac{(d / 2)^{\alpha}}{R}=4\left(\frac{d}{2}\right)^{\alpha}\left(\frac{2}{R_{\text {tot }}}\right)^{\frac{1}{4}} .
$$

For comparison, for a four-hop connection with hops of length $d / 4$, we have $R_{\text {tot }}=4 R^{\prime}$ and

$$
E_{\mathrm{tot}}^{\prime}=4\left(\frac{d}{4}\right)^{\alpha} \frac{4}{R_{\mathrm{tot}}}=2^{4-\alpha}\left(\frac{d}{2}\right)^{\alpha} R_{\mathrm{tot}}^{-1} .
$$

The ratio between the two energies is

$$
\frac{E_{\text {tot }}}{E_{\text {tot }}^{\prime}}=2^{\alpha-\frac{7}{4}} R_{\text {tot }}^{\frac{3}{4}} .
$$

Hence, the diversity scheme is more efficient for

$$
R_{\text {tot }}<2^{\frac{-4 \alpha+7}{3}} \text { or } p_{\mathrm{D}}>\exp \left(-2^{\frac{-4 \alpha+7}{3}}\right) \text {. }
$$

This curve is plotted in Fig. 9(a). Substantial energy gains are possible for high $p_{\mathrm{D}}$ [see Fig. 9(b)]. When the path loss exponent increases by one, the energy gain decreases by $3 \mathrm{~dB}$.

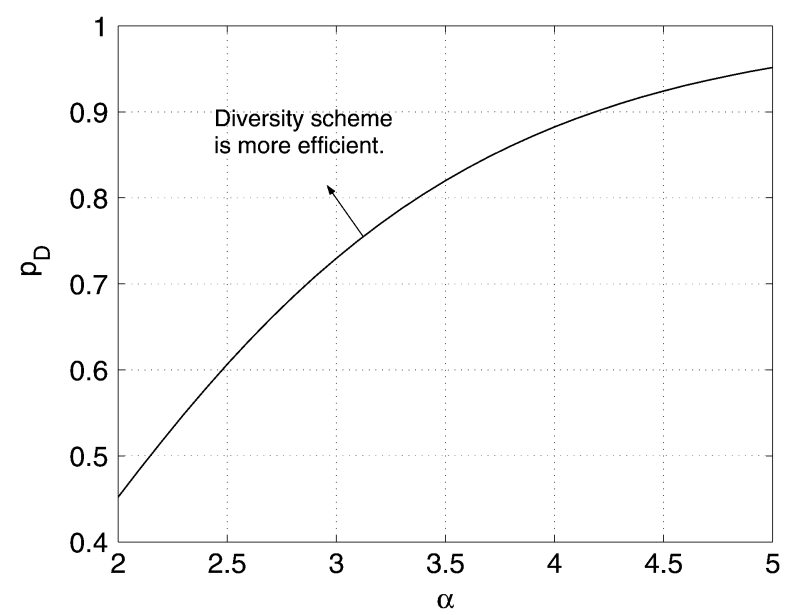

(a)

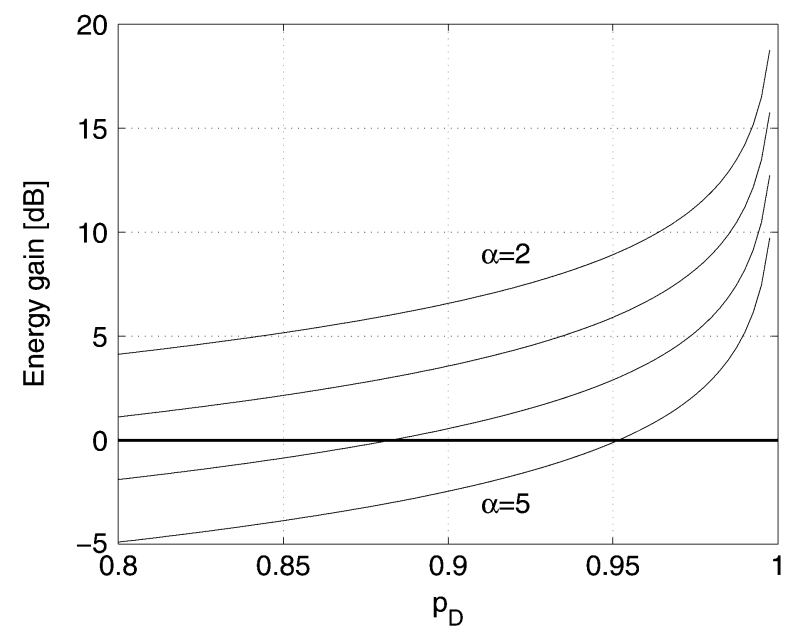

(b)

Fig. 9. (a) Region in the $\left(\alpha, p_{\mathrm{D}}\right)$ plane where the diversity scheme outperforms conventional multihop routing. (b) Energy gain as a function of the end-to-end probability.

This diversity scheme can be generalized to clusters of size $m$ that transmit over $n$ hops. In this case, $R_{\text {tot }}=n R^{m^{2}}$ and

$$
E_{\mathrm{tot}}=m n\left(\frac{d}{n}\right)^{\alpha}\left(\frac{n}{R_{\mathrm{tot}}}\right)^{\frac{1}{m^{2}}} .
$$

For the multihop scheme with $m n$ hops, ${ }^{5} R_{\text {tot }}=m n R^{\prime}$ and

$$
E_{\mathrm{tot}}^{\prime}=m n\left(\frac{d}{m n}\right)^{\alpha} \frac{m n}{R_{\mathrm{tot}}} .
$$

The ratio is

$$
\frac{E_{\mathrm{tot}}}{E_{\mathrm{tot}}^{\prime}}=R_{\mathrm{tot}}^{1-\frac{1}{m^{2}}} n^{\frac{1}{m^{2}}-1} m^{\alpha-1}
$$

from which we see that the energy gain is maximized for $m=2$ (except for $\alpha=2$, where $m=3$ performs slightly better) and increases almost linearly in $n$. We conclude that for high $p_{\mathrm{D}}$ and $\alpha$ not too big, the diversity scheme clearly outperforms conventional multihop routing.

\footnotetext{
${ }^{5}$ This comparison is fair both in terms of the number of nodes involved and
} in the delay, since the total number of transmissions is $m n$ for both schemes. 


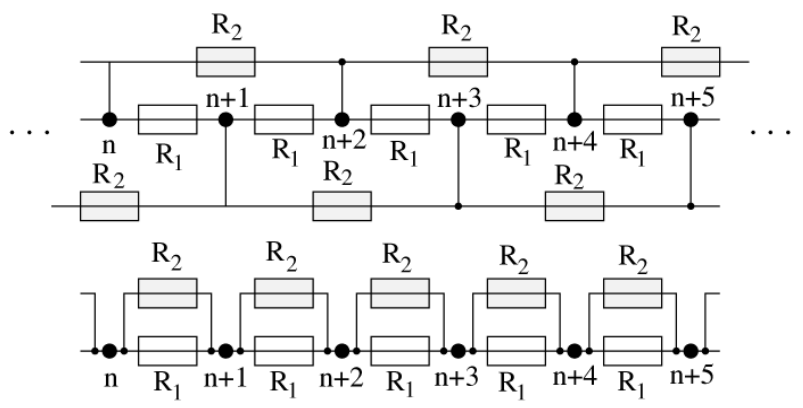

Fig. 10. (Top) Six nodes of a two-level leap-frog scheme. Every nodes listens to the transmissions of the two previous nodes. (Bottom) A conservative simplified erristor circuit.

Example 6. Long Line Networks with Equidistant Nodes: Consider a long line network with equidistant nodes of distance $d=1$, with the source at the left end and the destination at the right end. Every node transmits once but listens to the transmissions of $m$ of its left neighbors, resulting in $m-1$ implicit transmissions. The corresponding erristor circuit for $m=2$ is shown in Fig. 10. Due to the long chain of dependencies, such a leap-frog scheme ${ }^{6}$ is difficult to analyze; we, therefore, simplify it by attaching the implicit transmission to node $n+1$ rather than node $n$ (Fig. 10). This yields an upper bound on the erristance, since the accumulated erristance at node $n$ is certainly smaller than at node $n+1$. The per-hop erristance $R$ is then

$$
R=R_{1} \cdot 2^{\alpha} R_{1} \cdot 3^{\alpha} R_{1} \cdot \ldots \cdot m^{\alpha} R_{1}=R_{1}^{m}(m !)^{\alpha} .
$$

The choice $m=\left\lfloor R_{1}^{-1 / \alpha}\right\rfloor$ ensures that nodes do not listen to transmissions with low reliability. The benefit compared with conventional multihop routing is

$$
\frac{R_{1}}{R}=R_{1}^{1-m} \cdot(m !)^{-\alpha}
$$

which is enormous, in particular at high SNR (low $R_{1}$ ), as it is illustrated in Fig. 11 for $R_{1}=\left[10^{-3}, 0\right]$. As $2^{\alpha} R_{1}$ is larger than one, the benefit predicted from the erristor framework vanishes, whereas a more accurate analysis based on actual probabilities would still yield a gain. If the receive energy is taking into account, however, it may not be worthwhile to spend energy on listening to remote transmitters, since the incremental gain may be comparatively small. Therefore, we only consider transmitters with erristances smaller than one, and we limit $m$ to 5 .

Finally, to demonstrate the applicability of the erristor concept to random networks, let us consider line networks with uniformly random node distribution.

Example 7. Random Line Networks: For long line networks with uniformly random distribution, the internode distances $D_{k}$ are exponentially distributed and independent identically distributed (i.i.d.), and without loss of generality, we assume unit mean. For unit transmit power $(P=1)$, the erristance equals $D_{k}^{\alpha}$, and its pdf is

$$
p_{R}(r)=\exp \left(-r^{\frac{1}{\alpha}}\right) \frac{r^{\frac{1}{\alpha}}}{\alpha r} .
$$

${ }^{6}$ This name is borrowed from analog RLC filter design.

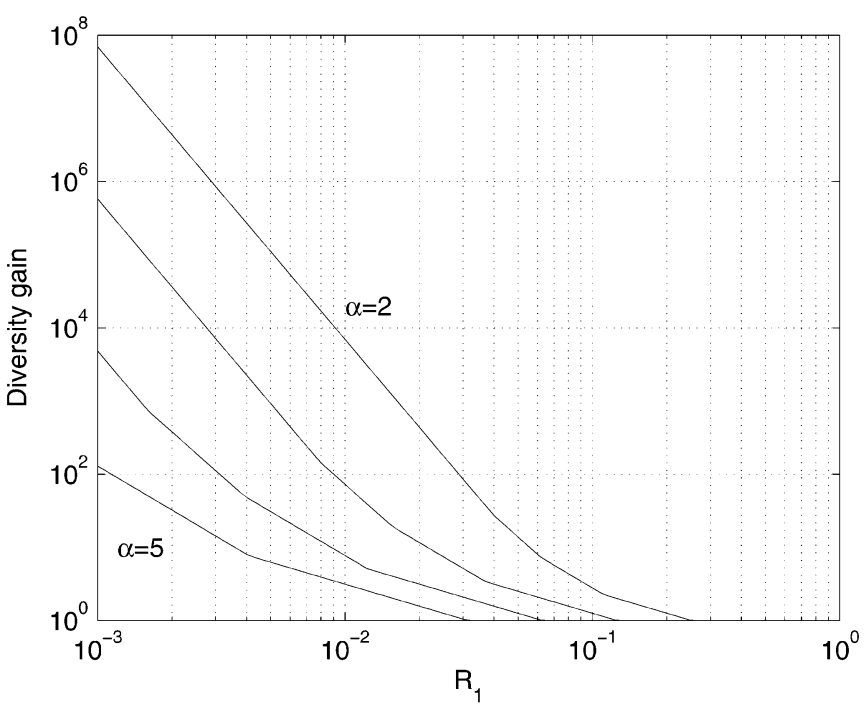

Fig. 11. Diversity gain from listening to $m$ transmissions as a function of the single-transmission erristance $R_{1}$ for $\alpha=2,3,4,5 . m=\min \left\{5,\left\lfloor R_{1}^{-1 / \alpha}\right\rfloor\right\}$.

The mean of $R$ is the $\alpha$ th moment of an exponential random variable (RV), so we have $\mathbb{E}[R]=\Gamma(\alpha+1)$ or, if $\alpha$ is an integer, $\mathbb{E}[R]=\alpha$ !. For $P \neq 1$, this is to be divided by $P$.

For a two-neighbor cooperative scheme $(m=2)$, the expected per-hop erristance is $\bar{R}_{2}=\mathbb{E}\left[D_{1}^{\alpha}\left(D_{1}+D_{2}\right)^{\alpha}\right]$, and in general, with transmit power $P$, we have

$$
\bar{R}_{m}=\mathbb{E}\left[\prod_{j=1}^{m} R_{j}\right]=\mathbb{E}\left[\prod_{j=1}^{m} \frac{1}{P}\left(\sum_{k=1}^{j} D_{k}\right)^{\alpha}\right] .
$$

Since $D_{k}$ is i.i.d., this can be simplified to sums and products of higher moments of $D_{k}$, which were determined above. Fig. 12 shows the gain $\bar{R}_{1} / \overline{R_{m}}$ for $m=2$ and $m=3$. The gain increases with $P^{m-1}$, and it is seen that the higher the path loss exponent, the higher the SNR has to be in order to get a benefit.

The minimum $P$ necessary to see a benefit can be derived analytically. For $\alpha=m=2$, e.g., we find $\left(\mathbb{E}\left[D^{4}\right]+2 \mathbb{E}\left[D^{3}\right] \mathbb{E}[D]+\right.$ $\left.\mathbb{E}\left[D^{2}\right]^{2}\right) / P^{2}<\mathbb{E}\left[D^{2}\right] / P$, or $40 / P^{2}<2 / P$, which holds for $P>20$, in accordance with Fig. 12(a).

\section{CONCLUDING REMARKS}

The erristor formalism permits the mapping of unwieldy probability expressions into a simple circuit-like framework, which greatly simplifies the analysis and design of transmit schemes that are based on selection combining with time diversity, path diversity, or a combination thereof. The wireless multicast advantage is incorporated by implicit erristors. The erristor circuit is topologically equivalent to the network graph and can, therefore, be drawn in a straightforward manner. Resource (re)allocation problems can effortlessly be solved by simple arithmetic, which makes a real-time implementation possible. A series of examples demonstrated not only the capabilities of this analysis and design tool, but also the huge gain that is possible from diversity-based communication strategies, in particular when high reliabilities are required. With more complex combining techniques, such as maximum ratio combining, this gain could be improved even further. 


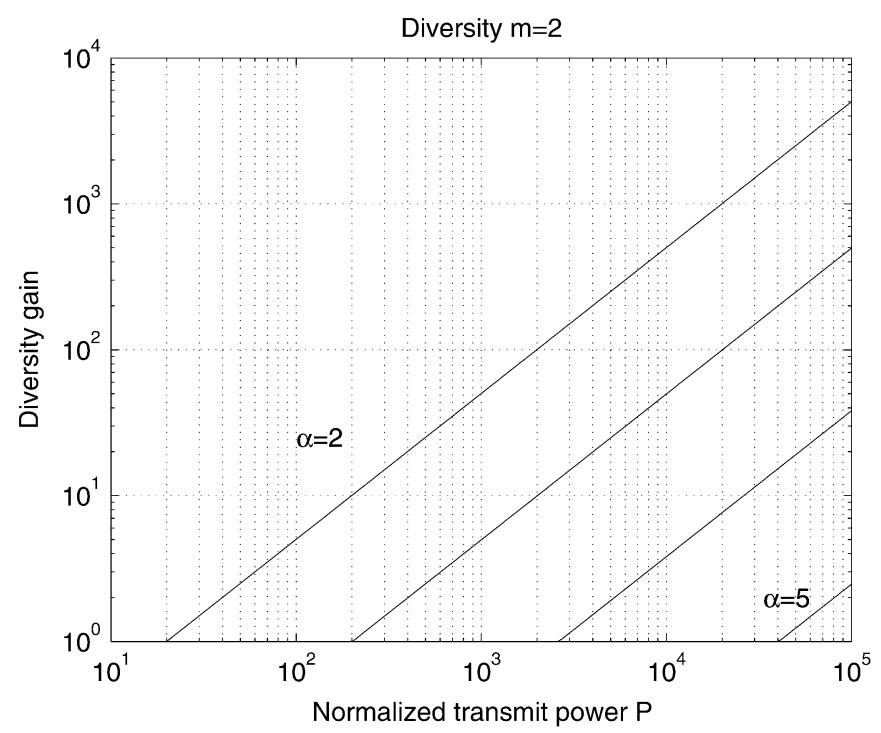

(a)

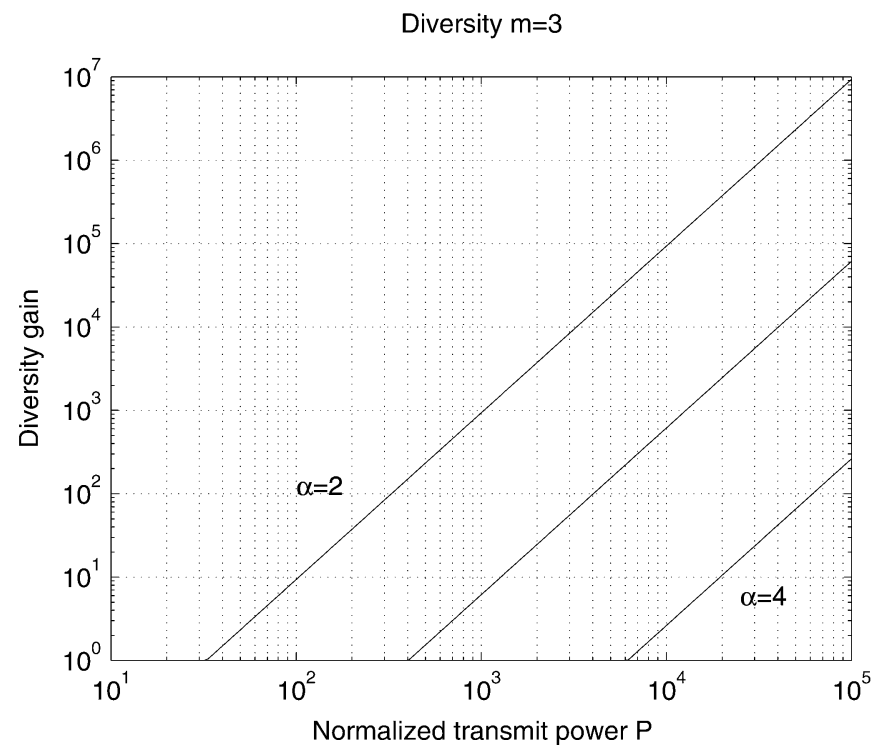

(b)

Fig. 12. Diversity gain for random networks as a function of the normalized transmit power $P$ for $\alpha=2,3,4,5$. (a) $m=2$. (b) $m=3$.

Further, the formalism is useful for educational purposes, since the multiplication property of parallel erristors impressively demonstrates the benefits of diversity schemes, and the series connection shows how the noise and, in turn, the packet loss probability accumulate over multiple hops.

The simple multinomial expressions for end-to-end erristances also lend themselves to a sensitivity analysis, i.e., the determination of the sensitivity of the total erristance to the individual erristances $\partial R_{\mathrm{tot}} / \partial R_{i}$. This permits the allocation of power where it has the most impact.

Erristor circuits are relatives of both electronic circuits and signal flow graphs (SFGs), but some important differences should be pointed out. The analogy to electronic resistive circuits only holds for serial elements; the multiplication of parallel erristors does not have a counterpart in electric circuits. Further, the erristor diagram is directed, it requires information about the direction of the transmission, i.e., who is transmitting and who is receiving. To make this clear, an arrow could be used as in SFGs to indicate direction. This would also add timing information to the diagram, since it would indicate a natural sequence of the transmissions.

Compared with SFGs, the way series and parallel branches are treated is different. In an SFG, transmittances of serially connected branches are multiplied and parallel branches result in addition, whereas in the erristor diagram, serial branches are added and parallel branches are multiplied. If instead of NSRs, SNRs were used, the multiplication of serial branches would hold for both graphs, but $k$ parallel branches would have to be treated like parallel resistors, yielding the harmonic mean divided by $k$. Further, the topology of the SNR-based circuit would no longer be identical to the original ad hoc network.

\section{APPENDIX \\ PROOF OF THEOREM 2}

Proof: In the inequality [see (11)]

$$
1-\prod_{i=1}^{n}\left(1-e^{-x_{i}}\right) \geq e^{-\prod_{i=1}^{n} x_{i}}
$$

it is easy to see that equality holds (both sides are equal to 1 ) if one of the $x_{i}$ is zero. It remains to show that $f_{n}(\cdot): \mathbb{R}^{n} \rightarrow \mathbb{R}$

$$
f_{n}\left(x_{1}, x_{2}, \ldots, x_{n}\right)=1-\prod_{i=1}^{n}\left(1-e^{-x_{i}}\right)-e^{-\prod_{i=1}^{n} x_{i}}
$$

is positive if all $x_{i}$ are positive. We note that $f$ goes to zero if $\forall i, x_{i} \rightarrow \infty$. Hence, $f$ is positive for positive $x_{i}$ if its partial derivatives $\partial f / \partial x_{i}$ are positive at zero and have at most one zero for positive $x_{i}$. Since the function is symmetric in all $x_{i}$, it is sufficient to consider only one partial derivative. An inductive technique is employed, discussing the case $n=2$ first.

Consider

$$
g_{2}(x):=f_{2}(x, y)=e^{-x}+e^{-y}-e^{-x-y}-e^{-x y}
$$

for a fixed $y \geq 0$. For $y=0, g_{2}(x) \equiv 0$, and for $y>0$, we note that $g(0)=0$ and $\lim _{x \rightarrow \infty} g(x)=e^{-y}>0$. Since

$$
g^{\prime}(x)=\frac{d g}{d x}=e^{-x}\left(-1+e^{-y}+y e^{x(1-y)}\right)
$$

there exists a single local extremum $\bar{x}$ for $y>0$ at

$$
\bar{x}=\frac{1}{y-1} \ln \left(\frac{y}{1-e^{-y}}\right) .
$$

As $y>\left(1-e^{-y}\right)$ for $y>0$, we find $\bar{x}>0$ for $y>1$ and $\bar{x}<0$ for $0<y<1$. For $y=1$, no solution exists, and for $y=0, g^{\prime}(x) \equiv 0$. Since $g^{\prime}(0)=-1+e^{-y}+y>0$, it is clear that the extremum is a maximum. So, we have $g(0)=0$, 
and for $0<y \leq 1, g(x)$ is monotonically increasing, whereas for $y>1$, it is monotonically increasing up to $\bar{x}$, and then monotonically decreasing to $e^{-y}>0$. Hence, $g(x)>0$ for $x>0$, and we have proven the theorem for $n=2$.

Now, assuming it is true for $n-1$, we prove the induction step.

For the general function $g_{n}(x):=f\left(x, x_{2}, \ldots, x_{n}\right)$ with fixed $x_{i} \geq 0$ for $i>1$, we note that $g_{n}(0)=0$ and

$$
\lim _{x \rightarrow \infty}=1-\prod_{i=2}^{n}\left(1-e^{-x_{i}}\right) \geq 0 .
$$

With $Q:=\prod_{i=2}^{n} x_{i}$, we get

$$
g_{n}^{\prime}(x)=e^{-x}\left(-\prod_{i=2}^{n}\left(1-e^{-x_{i}}\right)+Q e^{x(1-Q)}\right)
$$

Evaluation at $x=0$ yields

$$
\begin{aligned}
g^{\prime}(0) & =Q-\prod_{i=2}^{n}\left(1-e^{-x_{i}}\right) \\
& \geq 1-e^{-Q}-\prod_{i=2}^{n}\left(1-e^{-x_{i}}\right) \\
& =f_{n-1}\left(x_{2}, x_{3}, \ldots, x_{n}\right) \\
& \geq 0
\end{aligned}
$$

where we have made use of the induction. Again, equality holds for $Q=0$ only. Solving $g_{n}^{\prime}(\bar{x})=0$ yields the single extremum

$$
\bar{x}=\frac{1}{Q-1} \ln \left(\frac{Q}{\prod_{i=2}^{n}\left(1-e^{-x_{i}}\right)}\right) .
$$

We already established in (37) that the numerator is greater than (or equal to) the denominator in the logarithm, so, analogously to (34), we find that $\bar{x}<0$ for $0<Q<1$ and $\bar{x}>0$ for $Q>1$. Again, no solution for $Q=1$ and $g^{\prime}(x) \equiv 0$ for $Q=0$.

\section{REFERENCES}

[1] V. Rodoplu and T. H. Meng, "Minimum energy mobile wireless networks," IEEE J. Sel. Areas Commun., vol. 17, no. 8, pp. 1333-1344, Aug. 1999.

[2] A. Ephremides, "Energy concerns in wireless networks," in IEEE Wireless Commun., vol. 9, Aug. 2002, pp. 48-59.

[3] A. J. Goldsmith and S. B. Wicker, "Design challenges for energy-constrained ad hoc wireless networks," in IEEE Wireless Commun., vol. 9 , Aug. 2002, pp. 8-27.

[4] D. Tse and S. Hanly, "Multi-access fading channels: Part I: Polymatroid structure, optimal resource allocation and throughput capacities," IEEE Trans. Inf. Theory, vol. 44, no. 7, pp. 2796-2815, Nov. 1998.

[5] P. Viswanath, D. Tse, and R. Laroia, "Opportunistic beamforming using dumb antennas," IEEE Trans. Inf. Theory, vol. 48, no. 6, pp. 1277-1294, Jun. 2002.

[6] J. N. Laneman, D. N. C. Tse, and G. W. Wornell, "Cooperative diversity in wireless networks: Efficient protocols and outage behavior," IEEE Trans. Inf. Theory, to be published.

[7] J. A. Silvester and L. Kleinrock, "On the capacity of multihop slotted ALOHA networks with regular structure," IEEE Trans. Commun., vol. COM-31, no. 8, pp. 974-982, Aug. 1983.
[8] H. Takagi and L. Kleinrock, "Optimal transmission ranges for randomly distributed packet radio terminals," IEEE Trans. Commun., vol. COM-32, no. 3, pp. 246-257, Mar. 1984.

[9] L. Hu, "Topology control for multihop packet networks," IEEE Trans. Commun., vol. 41, no. 10, pp. 1474-1481, 1993.

[10] J. L. Wang and J. A. Silvester, "Maximum number of independent paths and radio connectivity," IEEE Trans. Commun., vol. 41, no. 10, pp. 1482-1493, Oct. 1993.

[11] M. Sanchez, P. Manzoni, and Z. Haas, "Determination of critical transmission range in ad-hoc networks," in Proc. Multiaccess, Mobility, Teletraffic Wireless Commun. (MMT'99), Venice, Italy, Oct. 1999.

[12] P. Gupta and P. R. Kumar, "The capacity of wireless networks," IEEE Trans. Inf. Theory, vol. 46, no. 2, pp. 388-404, Mar. 2000.

[13] M. Grossglauser and D. Tse, "Mobility increases the capacity of ad-hoc wireless networks," in Proc. IEEE INFOCOM, Anchorage, AL, 2001.

[14] G. Németh, Z. R. Turányi, and A. Valkó, "Throughput of ideally routed wireless ad hoc networks," ACM Mobile Comput. Commun. Rev., vol. 5 , no. 4, pp. 40-46, 2001.

[15] C. Schurgers, V. Tsiatsis, S. Ganeriwal, and M. Srivastava, "Optimizing sensor networks in the energy-latency-density design space," IEEE Trans. Mobile Comput., vol. 1, no. 1, pp. 70-80, 2002.

[16] T. J. Shepard, "A channel access scheme for large dense packet radio networks," presented at the ACM SIGCOMM, Stanford, CA, Aug. 1996, [Online]. Available: http://www.acm.org/sigcomm/sigcomm96/ papers/shepard.ps.

[17] E. S. Sousa and J. A. Silvester, "Optimum transmission ranges in a direct-sequence spread-spectrum multihop packet radio network," IEEE J. Sel. Areas Commun, vol. 8, no. 5, pp. 762-771, Jun. 1990.

[18] D. A. Maltz, J. Broch, and D. B. Johnson, "Lessons from a full-scale multihop wireless ad hoc network testbed," IEEE Pers. Commun., vol 8, no. 1, pp. 8-15, Feb. 2001.

[19] D. Ganesan, B. Krishnamachari, A. Woo, D. Culler, D. Estrin and S. Wicker. (2002) An empirical study of epidemic algorithms in large scale multihop wireless networks. Intel Res. Rep. IRB-TR-02-003. [Online]. Available: www.intel-research.net/Publications/Berkeley/050220021 703_19.pdf

[20] T. S. Rappaport, Wireless Communications-Principles and Practice, 2nd ed. Englewood Cliffs, NJ: Prentice-Hall, 2002.

[21] M. Zorzi and S. Pupolin, "Optimum transmission ranges in multihop packet radio networks in the presence of fading," IEEE Trans. Commun. vol. 43, no. 7, pp. 2201-2205, Jul. 1995.

[22] M. Haenggi, "Probabilistic analysis of a simple MAC scheme for ad hoc wireless networks," in IEEE CAS Workshop Wireless Commun. Netw. Pasadena, CA, Sep. 2002.

[23] X. Liu and M. Haenggi, "Throughput bounds and energy consumption of mobile multihop networks," in Proc. IEEE Veh. Technol. Conf. (VTC'04 Fall), Los Angeles, CA, Sep. 2004.

[24] J. N. Laneman and G. W. Wornell, "Energy-efficient antenna sharing and relaying for wireless networks," in Proc. IEEE Wireless Commun. Netw. Conf. (WCNC'O0), Chicago, IL, 2000, pp. 7-12.

[25] J. E. Wieselthier, G. D. Nguyen, and A. Ephremides, "On the construction of energy-efficient broadcast and multicast trees in wireless networks," in Proc. IEEE INFOCOM, Tel-Aviv, Israel, Mar. 2000, pp. 585-594.

[26] P. Gupta and P. R. Kumar, "Toward an information theory of large networks: An achievable rate region," IEEE Trans. Inf. Theory, vol. 49, no. 8, pp. 1877-1894, Aug. 2003.

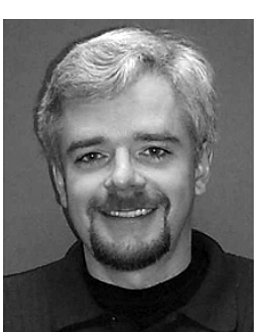

Martin Haenggi (S'96-M'99-SM'04) received the Dipl. Ing. (M.Sc.) degree in electrical engineering, the Dipl. NDS ETH (post-diploma) degree in information technology, and the Ph.D. degree from the Swiss Federal Institute of Technology (ETHZ), Zurich, Switzerland, in 1995, 1996, and 1999, respectively. His Ph.D. dissertation was on the analysis, design, and optimization of cellular neural networks.

In 1995, he joined the Signal and Information Processing Laboratory, ETHZ, as a Teaching and Research Assistant. After a postdoctoral year at the Electronics Research Laboratory, University of California, Berkeley, he joined the faculty of the Department of Electrical Engineering, University of Notre Dame, Notre Dame, IN, as an Assistant Professor in January 2001. His scientific interests include networking and wireless communications, with an emphasis on ad hoc and sensor networks.

Dr. Haenggi was awarded the ETH Medal for both his M.Sc. thesis and Ph.D. dissertation. He received the 1995 Willi Studer Award at ETH. 